\title{
Quelles marginalités ? La recherche de la différence chez quelques écrivains à la charnière des $\mathrm{XX}^{\mathrm{e}}$ et $\mathrm{XXI}^{\mathrm{e}}$ siècles
}

Pierre Laroche

\section{(2) OpenEdition \\ 12 Journals}

Édition électronique

URL : http://journals.openedition.org/cei/916

DOI : 10.4000/cei.916

ISSN : 2260-779X

Éditeur

UGA Éditions/Université Grenoble Alpes

Édition imprimée

Date de publication : 15 mai 2008

Pagination : 125-135

ISBN : 978-2-84310-121-2

ISSN : 1770-9571

Référence électronique

Pierre Laroche, «Quelles marginalités ? La recherche de la différence chez quelques écrivains à la charnière des XXe et XX|e siècles », Cahiers d'études italiennes [En ligne], 7 | 2008, mis en ligne le 15 novembre 2009, consulté le 19 avril 2019. URL : http://journals.openedition.org/cei/916 ; DOI $10.4000 /$ cei. 916 


\section{QUELLES MARGINALITÉS? \\ LA RECHERCHE DE LA DIFFÉRENCE CHEZ QUELQUES ÉCRIVAINS À LA CHARNIĖRE DES XXe ET XXI" SIĖCLES}

Pierre Laroche

Université de la Sorbonne Nouvelle-Paris 3

Les questions examinées ici portent sur un certain nombre de textes narratifs de la fin du XX ${ }^{\mathrm{e}}$ siècle, pour l'essentiel des œuvres d'écrivains momentanément identifiés comme "nuovi cannibali», ceux qui figurent dans l'anthologie Gioventù cannibale, la prima antologia italiana dell'orrore estremo $^{1^{*}}$, notamment Niccolò Ammaniti, Carlo Lucarelli, Aldo Nove. Ces écrivains ne se sont pas limités à leur contribution à cette anthologie et on note d'entrée de jeu que nombre des titres de leurs romans ou nouvelles, Gioventù cannibale, antologia italiana dell'orrore estremo, Vivere e morire al Prenestino, L'ultimo capodanno dell'umanità, Ti sogno con terrore, Il giorno del lupo, Lupo mannaro, Serial Killer - Storie di ossessione omicida, Il trillo del diavolo, Fa un po'male, annoncent une esthétique de la violence sadique, jouant sur les registres de la peur, du cynisme, de l'ironie, de l'humour macabre 2 .

Ces auteurs ont été "momentanément» identifiés comme «nuovi cannibali» d'abord parce que, comme le dit Raffaele Manica, «il y a peu de survivants de la célèbre Gioventù cannibale ${ }^{3}$ ». En même temps, leur évolution les éloigne bientôt de certaines tendances extrêmes du «cannibalisme» : on ne trouve dans Io non ho paura ${ }^{4}$ d'Ammaniti ni les fantasmes sexuels ni la violence sanguinolente de $F a$ un po'male qui va chez certains auteurs jusqu'à l'anthropophagie ${ }^{5}$. Ce qui n'empêche qu'il serait sans doute possible de rechercher dans Io non ho paura ce qui perdure des structures narratives des "cannibales", de la maîtrise du suspense (y compris par la fin ouverte) qui substitue la terreur à l'horreur, ou, dans Guernica $^{6}$ de Lucarelli, où l'on pourrait attendre une œuvre politique, la dimension onirique et fantastique constante et même dominante dans le finale.

\footnotetext{
* Notes p. 134.

Cahiers d'études italiennes, $\mathrm{n}^{\circ}$ 7, 2008, p. 125-135. 125
} 
À ces "cannibales», j'ai joint Enrico Brizzi, dont le roman Jack Frusciante $^{7}$ è uscito dal gruppo $(1994)^{8}$ n'a en commun avec eux ni la violence ni la sexualité envahissante dans le récit et l'expression. Au contraire, les amours d'Alex et Aidi ${ }^{9}$ sont parfaitement chastes et très "fleur bleue", ce qui donne lieu à des images d'une fraîcheur étonnante compte tenu de la tonalité générale du corpus ici examiné: "gli sorrideva come un'alba d'inverno" (p. 23); on trouve aussi chez Brizzi une invention lexicale débridée qui dépasse ce qu'on trouve chez les «cannibales».

De façons diverses, les personnages de ces romans ou récits peuvent être considérés comme relevant de la marginalité. Pas telle qu'on l'entend couramment, celle qui touche des personnes rejetées hors de la société, parfois conduites à des formes variées de délinquance, sans domicile, sans travail, parfois sans famille, généralement pour des raisons sociales, économiques, politiques, rarement par un choix ou un consentement délibérés de leur part, et encore faudrait-il comprendre ce qui les a conduites ou poussées à ce choix ou à ce consentement. Pas, non plus, la marginalité qui est le lieu social, économique, politique et culturel de certaines œuvres des années trente, comme Tre operai de Carlo Bernari, Berlin Alexanderplatz de Döblin, ou la trilogie de Roberto Arlt, El juguete rabioso, Los siete locos et Los lanzallamas. Ces dernières œuvres ont pour protagonistes des adultes qui ont un rapport avec la société, tentative d'intégration, opposition, volonté de destruction, et une sorte de projet politique, l'utopie d'une autre société, projet plus ou moins conscient, plus ou moins fantasmé, fondé sur le refus de la société où ils vivent, refus dont il faut reconnaître qu'il ne se place pas toujours dans une perspective humaniste et libératrice. La marginalité est peut-être ici la forme la plus forte de la différence, définie en fonction d'un regard sur l'ensemble de la société, ses inégalités, ses conflits, les règles qui assurent sa reproduction.

Les personnages des textes narratifs italiens qui sont traités ici sont des jeunes, jeunes adultes, adolescents, parfois des enfants ${ }^{10}$. On ne sait pas avec précision à quel moment ont lieu les faits racontés, mais on peut les situer à la fin du siècle dernier ${ }^{11}$. On ne peut dire si ces jeunes sont heureux ou malheureux; Brizzi écrit de son personnage Alex: "si sentiva profondamente infelice ma in modo distaccato [...] come se la sua vita appartenesse a qualcun altro» (Jack Frusciante..., p. 11). Daniele Brolli voit en eux des «individus sans désirs ni conscience ${ }^{12}$ ». On ne sait rien ou peu de choses de leur famille, souvent absente, qu'ils méprisent ou massacrent, de leurs activité et statut sociaux, mais, en général, ils sont de 
milieux assez aisés. Ils sont socialement et économiquement indifférenciés, à part quelques exceptions: dans Il rumore, le narrateur rapporte des faits qu'il a commis dans un milieu défavorisé, "un quartiere popolare a Cologno Monzese (per chi non lo sapesse, Cologno era, ed è tuttora, un sobborgo-dormitorio alle porte di Milano)» (p. 126-127). La parenthèse «è tuttora» ramène cette histoire à l'actualité, comme le cauchemar que le narrateur essaie d'exorciser par son récit. Mais les faits narrés remontent à la fin des années cinquante, quand il avait dix ans; il déclare maintenant: «non mi posso lamentare. Faccio il redattore in una rivista femminile, guadagno abbastanza bene da mantenere la mia famiglia", toutes choses qui l'amènent à ne mentionner que comme contexte de la délinquance des autres $^{13}$ les conditions socio-culturelles dans lesquelles il a commis une agression sexuelle qu'il ne parvient pourtant pas à censurer et qui est la clé du récit; mais la contiguïté de la narration de cet acte avec celle des violences familiales conduit évidemment le lecteur à établir une responsabilité de ces conditions que le narrateur donne l'impression de ne pas vouloir retenir.

La sexualité, sous des formes violentes, agressions, actes de sadisme, viols, mutilations et auto-mutilations, est omniprésente dans ces œuvres. La violence y conduit souvent au meurtre. De façon générale, la mort rôde en permanence. Cadia Farinato relève la récurrence des termes liés au sang, à la couleur rouge et à la mort chez Ammaniti et Aldo Nove ${ }^{14}$. En revanche, d'autres formes de délit, le vol, l'escroquerie, la fausse monnaie, les excès de vitesse, sont rares ou inexistantes. Certes, Martino, l'ami d'Alex, son modèle dans Jack Frusciante..., est mêlé au trafic de la drogue; dans Fa un po'male, Aldo et Robbi volent un bébé kangourou dans un zoo. Mais, dans l'ensemble, ils font penser à ce qu'écrivait Patrick Kéchichian rendant compte de Les mauvestis de Frédéric Valabrègue ${ }^{15}$ :

quelques adolescents ou jeunes adultes. Pas des marginaux ou des voyous, juste des jeunes au seuil de la vie autonome, ou la commençant. Et qui savent déjà qu'ils ne referont jamais le monde, qu'au contraire tout est réuni pour que leur départ dans la vie n'ait rien de joyeux, de rose ${ }^{16}$.

Cependant, même s'il n'y a pas vraiment de projet concret, il y a chez Brizzi l'expression d'une sorte de nostalgie de cette période où une volonté de changement s'est exprimée en Italie, comme dans d'autres pays européens dans les années soixante-dix, souvenir d'enfance car il «vecchio Alex ${ }^{17}$ " n'avait pas dix ans à cette époque. Il se définit comme "catholic punk» ou «red catholic» (p. 79), ne supporte pas «i ciellini ${ }^{18}$ », raille «le cinquantenni stronze che piangevano in estasi facendo tinitinnare $\mathrm{i}$ gioielli e sbavandosi il trucco [...] le facce di culo ipocrite che incontrava 
in chiesa. Compresi i giovani del catechismo» (p. 49); il souhaite que soit construite "una nuova Italia perché la Prima Repubblica era fallita» (p. 107), s'indigne de l'assassinat du juge Giovanni Falcone ${ }^{19}$. Ses compagnons les plus proches

parlavano anche di politica [...] si sentivano rossi, radicali, anarchici. Odiavano, ricambiati, gli stronzi nazisti che proliferavano nella loro scuola $[\ldots]$; si sentivano parte, con sfumature differenti, d'una sinistra sorridente e sincera; simpatizzavano con il mondo underground dei centri sociali occupati e delle case discografiche indipendenti, e soprattutto odiavano i pinocchi di piombo delle organizzazioni di partit ${ }^{20}$. (p. 57)

Mais Alex fait figure d'exception: les autres personnages de ces récits ne sont porteurs d'aucun projet politique, individuel ou collectif, même vague. Dans La merda, Aldo Nove le fait dire explicitement au narrateur dans une phrase où le dernier mot, inachevé, traduit l'incapacité assumée de formuler un tel projet: «Bisogna cambiare la situazione politica. Fare qualcosa per questo mondo. Lo pensavo sempre, da bambino. Oggi, ritengo $\mathrm{ch}^{21}$ »; de façon générale, Superwoobinda laisse l'image d'un monde où l'on n'a pas, où l'on n'a plus, ni rêve ni perspective: "La mia generazione ha tanto bisogno di sognare", regrette Aldo Nove (Woobinda, in Superwoobinda, p. 15). Même si le narrateur de Woobinda commente le faible niveau culturel des "televisioni di Berlusconi" en disant que "questo è uno degli effetti della destra», c'est pour une raison qu'on pourra juger subalterne: "non fanno più vedere Woobinda ${ }^{22}$, il ragazzo svizzero che correva nella savana ${ }^{23}$ ". Il y a cependant, chez certains personnages d'Aldo Nove, une nostalgie comparable à celle d'Alex, qu'on retrouve dans ce commentaire sur une émission de télévision: «Era un cartone animato di destra, un cartone animato della Lega Lombarda perché non aveva un discorso suo di fondo come Woobinda, che ci faceva sentire uniti quando uscivamo la sera a suonare i campanelli nel 1979 avevamo in mente quella cosa lì, ci faceva sentire uniti tutti, ora le forze sono $\mathrm{m}$ » (A. Nove, Woobinda, p. 16). Mais cette nostalgie n'a plus de charge polémique, n'est qu'un souvenir du bon vieux temps, elle s'exprime dans une phrase qui ne trouve pas sa fin, encore une fois. Dans l'ensemble, il paraît difficile de parler, comme le fait Cadia Farinato, d' «une forte critique sociale ${ }^{24}$ » : même chez Enrico Brizzi et Aldo Nove, on ne peut constater que le désenchantement, y compris chez les personnages qui expriment le plus d'insatisfaction face au fonctionnement de la société. Ainsi, dans Drammatico caso nel mondo dello sci, le frère du narrateur, mort en compétition, doit continuer à honorer un contrat qui l'oblige à représenter son sponsor jusqu'à la fin de la saison: "Mio fratello rappresenta il 
primo caso di morto che si classifica terzo a una prova mondiale. Grazie ai propulsori a ossigeno compresso trapiantati nella schiena, la sua velocità attuale non è mai scesa sotto i livelli che abitualmente raggiungeva da vivo" (in Superwoobinda, p. 74 -75); ici, l'humour dédramatise l'expression et pourrait lui donner plus d'objectivité, mais le passage à l'invraisemblable la prive de virulence au bénéfice d'une absurdité simplement comique.

On a donc surtout, du fait de l'apparente objectivité de récits dépassionnés, l'impression que les personnages ne se sentent pas concernés par ce qui les environne; ils n'ont souvent pas d'amis, tout au plus des compagnons de malheurs et de méfaits. De même que le héros de Brizzi «è uscito dal gruppo ", de même, dix ans plus tard, les élèves du protagoniste de Secoli di gioventù sont, selon les termes d'Eraldo Affinati, "fuori dal coro $^{25}{ }^{\prime}$. On pourrait dire qu'ils sont au moins autant des marginaux de la vie que des marginaux de la société. En rupture avec l'une et l'autre, il arrive qu'ils meurent d'une façon violente qui résulte de choix de vie qu'on pourrait appeler suicidaires mais qu'eux-mêmes n'expriment pas comme tels. Ainsi, les deux protagonistes d'Il mondo dell'amore ${ }^{26}$ meurent d'hémorragie parce qu'ils se sont émasculés, non pour mourir, mais afin de réaliser un fantasme, celui de devenir femmes "per provare una storia lesbo» (p. 61).

La différence doit être explicitée, par les autres, par soi-même, ou par les deux. Les protagonistes de ces œuvres se distinguent des autres par leur jeunesse. Jeunesse que leur reprochent ces autres, mais qu' ils revendiquent en exprimant leur haine, leur mépris, leur répugnance des "vieux». Dans Mia nonna ${ }^{27}$, le narrateur déteste sa grand-mère, il essaie de la tuer alors qu'elle est sur la tombe de son mari, mais «ovviamente non è morta [...]. Avrei dovuto considerare che giocava a casa»: ici la haine s'exprime dans cet humour macabre qui s'appuie sur ce qu'on pourrait appeler une idéologie du supporter. Ce qui motive cette haine, facilement identifiée à la haine des parents ${ }^{28}$, c'est que ceux-ci sont vus comme ayant la possibilité d'accéder à des biens qui paraissent inaccessibles et qu'éventuellement l'on méprise, par rejet des valeurs et des formes culturelles du monde des parents: Alex, visitant la chambre de son ami Martino, constate qu'il y a de quoi y rester des heures, à chercher le nom des personnages figurant sur les posters, à contempler la collection de vidéocassettes ${ }^{29}$, mais, pour ce qui est des livres, "sarebbe bastato un nanosecondo ${ }^{30}$ ». Et sa réflexion le conduit à une idée de la vie qui ne le pousse pas vers la recherche de nouveaux horizons: "le foto di Martino mi hanno dato la percezione reale della beffa: bastava stare fermi lì e cogliere l'occasione, kazzo... ${ }^{31}$ ». En con- 
séquence, outre les parents, les enseignants sont eux aussi la cible des sarcasmes d'Alex: «il conformismo e la doppiezza dei profii, il modo biforcuto che avevano di incoraggiare a parole l'indipendenza di giudizio dei ragazzi e la rabbia sottile con cui punivano ogni minimo segnale d'autonomia quei bastardi» (p. 12). La différence jeunes/vieux est donc une métaphore de la différence sociale et culturelle, entre pauvres et riches, ou tenus pour tels. La culture scolaire n'est d'ailleurs pas la seule à être tournée en dérision, la culture populaire l'est également, que ce soient les contes pour enfants, avec la parodie du Petit Chaperon rouge par Daniele Luttazzzi, ou la culture télévisuelle (voir Aldo Nove, Il mondo dell'amore), raillée au même titre que la société de consommation dans son ensemble.

Du coup, la haine ne s'adresse pas qu'aux vieux: Alex ne déteste pas seulement les adultes, mais aussi les jeunes qui ne sont pas de son clan, qui sont conformistes, les filles avec des bagues dentaires, les "semprevergini", les bons élèves qui rentrent à l'heure en classe, qui s'habillent à la mode, en somme ceux dont la vie est identique ou conforme à celle que leurs parents ont eue ou désirée. Dans cette différence qui tourne autour du rapport jeunes/vieux, les jeunes pensent que c'est le monde vieux qui est isolé: Aldo Nove parle du "desiderio spiazzante di essere sciolti in un unico, pulsante corpo che gode di avere 18 anni. La frigidità dell'aria del mondo era parcheggiata fuori dalla discoteca ${ }^{32} »$.

La volonté de rupture avec les générations précédentes s'exprime chez les auteurs dans le rapport à l'écriture, à la structure du récit, aux limites morales exprimées par la littérature reconnue comme telle dans l'Italie du siècle dernier. En ce qui concerne le récit, la différence s'exprime avant tout par la violence, violence physique, violence sexuelle, une violence démesurée qui coexiste avec la plus grande banalité de la vie courante. Cette violence s'exerce le plus souvent sans motif explicité: "des gestes dépourvus de passion et de sens, des actes qui déchirent le voile superficiel de la normalité pour révéler que ses bases reposent sur un terrain incandescent d'inquiétude»(D. Brolli, p. VII-VIII), contre les plus faibles, contre les vieux, contre les enfants, contre les femmes, montrées comme stupides, vulgaires, réduites à des objets de mépris et de domination, particulièrement de domination sexuelle, qu'elles soient consentantes ou non. Les "cannibales» auraient-ils pressenti avant d'autres la montée d'une violence gratuite, symptôme d'une société en perte de repères?

Cette différence entre les hommes jeunes et le reste des humains se traduit dans la fonction qu'ils occupent dans la narration : à part le narrateur protagoniste d'Il rumore, on ne voit guère une femme, un enfant ou un homme de plus de quarante ans protagoniste d'un récit des «cannibales». 
Violence verbale aussi, par une écriture qui vise à une rupture totale avec la tradition ${ }^{33}$ : déjà, chez Brizzi, sans doute sous l'influence de Pier Vittorio Tondelli, puis chez les "cannibales", l'écriture tente de restituer la langue orale des jeunes, multiplie interjections, onomatopées, abréviations, ellipses ${ }^{34}$, sigles, superlatifs, intercalaires, néologismes pouvant aller jusqu'à l'apax, américanismes, phrases brèves, tronquées qui calquent le style direct et laissent supposer une réflexion qui ne sait pas, ou n'ose pas aboutir, ou, au contraire, phrases très longues sans ponctuation, procédant par associations d'idées, à la limite de l'écriture automatique, le tout combiné avec des inflexions dialectales, des formes prises au langage populaire et à l'argot, au langage de la télévision et du journalisme, de la BD, de la publicité, une forte présence de la culture anglo-saxonne, nordaméricaine surtout. Pour ce qui est de l'écrit: orthographe inventée, ponctuation surabondante et un goût marqué pour les points de suspension et d'exclamation. On pourra objecter que tout cela n'est pas totalement nouveau, mais le côté systématique conduit Daniele Brolli à parler d'une «langue encore en voie de formation qui, sans fausse pudeur, récolte ses mots dans les programmes de télévision, la culture des rues, le cinéma, la musique pop ${ }^{35}$ ".

Les «cannibales» poursuivent sur cette voie, en généralisant le recours à la scatologie, au macabre, à l'obscénité: les organes génitaux des deux sexes sont abondamment mentionnés, sous leur dénomination argotique, avec des orthographes variées, tantôt avec leur sens propre, tantôt comme juron, tantôt comme appellatif ou insulte, voire comme simple intercalaire. Parfois, en revanche, l'écriture peut aussi être très recherchée et les références culturelles plus ou moins explicites ne sont pas rares ${ }^{36}$. Aldo Nove, dans sa nouvelle particulièrement sanguinolente Il mondo dell'amore (in Gioventù cannibale) conclut son récit par un texte de Nanni Balestrini et recourt même, pour l'épigraphe d'un chapitre, à une citation de Giacomo da Lentini: "Amor è uno desio che vien dal core per abundantia di gran plazimento e gli ogli in prima generan l'ardore e lo core gli dà nutrimento» (p. 58), juste avant une scène chirurgicale télévisée précédée de l'annonce suivante:

SI AVVERTONO GLI SPETTATORI FACILMENTE IMPRESSIONABILI DI ASTENERSI, DA QUESTO MOMENTO, DALLA VISIONE DELLE SEQUENZE SULLA SINTESI DELL'INTERVENTO CHIRURGICO SUL CAMBIAMENTO DI SESSO. (p. 59)

Ce n'est pas seulement l'aspect lexical et syntaxique qui est en cause: le rapport entre auteur, narrateur, protagonistes et lecteurs, est bouleversé. Tantôt Brizzi fait parler le protagoniste au style direct, tantôt il intervient 
en narrateur extra-diégétique, s'intègre à la fois au récit et au public, prend à témoin le lecteur pour s'auto-définir "quale conoscente del vecchio Alex" (Jack Frusciante..., p. 11); Lucarelli, dans Almost blue, procède de même, donne la fonction de narrateur soit à un personnage extra-diégétique soit à un protagoniste, pas toujours le même. La conduite du récit est ainsi remise en cause par des changements de narrateur imprévisibles, marqués à l'évidence par le langage cinématographique et surtout par la pratique du zapping devant la télévision.

À propos de l'influence du cinéma et de la télévision dans le récit et l'écriture, je reprends une analyse de Piero Cudini, qui, lors d'une réunion $\mathrm{du}$ "Centre interdisciplinaire de recherche sur la culture des échanges», le 12 mai 2001, observait qu'on a accordé peu d'importance au fait que, dans Jack Frusciante..., «le cinéma entre massivement dans le récit [...] le début même du roman de Brizzi "dépend" du cinéma; mais c'est un cinéma vu à la télévision": le nom des personnages, Aidi (Heidi), Alex (comme le héros du film de Kubrick, A Clockwork Orange), la place de la télévision dans la vie de leurs familles respectives, les références explicites à certains films (Il portaborse, Gone with the wind), le recours à un vocabulaire technique, la scène où Aidi quitte Alex pour aller étudier un an aux Etats-Unis (p. 170), scène que Piero Cudini qualifie de "pré-finale ouvertement filmique», soutiennent cette analyse ${ }^{37}$.

L'influence de la BD et du cartoon est également sensible, par exemple dans l'écriture riche en onomatopées et dans le mélange de réalisme et d'invraisemblable. On en a un exemple particulièrement frappant avec la fin de Cappuccetto Splatter, où Cappuccetto Rosso et le vieux ***, assassinés, découpés en dés mis au congélateur, passés à la moulinette, dévorés en partie, sont arrachés du ventre de leur assassin et en sortent parfaitement vivants, Cappuccetto Rosso s'exclamant "Cazzo. Non sai che incubo!» et concluant qu'elle ne sortira plus seule le soir dans les rues de Milan. C'est la même «logique» narrative, la complaisance macabre en moins, que celle des cartoons de Tex Avery et surtout de Hannah Barbera, où le coyote écrasé par une tonne de rochers ou laminé par un train réapparaît dans la séquence suivante, allumant allègrement la mèche d'une cartouche de dynamite. Dans la mesure où cette violence est vaine, dédramatisée, ne tue pas, on peut évidemment y voir la puérilité d'un fantasme d'immortalité, dont témoignent depuis longtemps certains contes comme Pierre et le loup. De façon plus générale, on peut juger invraisemblable le fait que les meurtres n'aient jamais de conséquences pour les meurtriers, ne fût-ce que parce que ceux-ci n'ont aucune difficulté à effacer les traces du crime, cadavres y compris. À quelques exceptions près, 
comme Almost blue de Lucarelli, ces textes ne tiennent aucun compte de la police; mais précisément, Almost blue est structuré comme un roman policier.

La télévision cristallise les contradictions de ces personnages: elle fait partie du décor de la vie, remplace le contact avec la société: dans La strage di via Palestro, le narrateur en fait le constat: «Ho vent'anni. Passando tra la gente, mi vedevo tra le macerie ed ero triste, ma meno che guardando la televisione, perché alla televisione, tutto sembra vero... ${ }^{38}$ ». Mais, ressentie comme le symbole de ce qui banalise l'horreur et la platitude conjointes, la vie violente et sans attrait qu'ils vivent, elle est aussi objet de dérision. Elle est une «odiosamata" qui s'intègre dans une réception contradictoire de la «modernité», qui est à la fois le monde où vivent ces jeunes et la société et le mode de vie créés par les adultes qu'ils rejettent. Chez Brizzi, ce refus du «moderne» est particulièrement sensible: Alex ne se déplace qu'à bicyclette mais sa référence en la matière n'est ni Pantani ni Fausto Coppi, probables héros de la génération de ses parents et grands-parents, mais Girardengo ${ }^{39}$.

Un des traits de cette littérature "cannibale» qui se veut fondamentalement différente de l'écriture et des formes narratives du passé est ainsi le contact entre divers héritages culturels et les formes les plus récentes de la communication, entre les apports de diverses formes d'expression culturelle et entre divers niveaux d'écriture. Plus que d'une littérature de rupture, on pourrait parler d'éclectisme qui juxtapose le dolce stil novo et la pornographie, la référence classique, voire archaïque, aux médias les plus récents et à la communication publicitaire.

D'un autre point de vue, concernant non plus l'écriture mais l'idéologie, on pourrait parler de littérature du conjoncturel, du précaire, de l'incertain et de l'inutile: il n'y a plus de certitudes, de projets, de rêve et d'utopie; il y en a eu, les personnages les connaissent, certains les ont vécus, y ont même cru et participé. Désormais, ils rejettent le monde actuel, et du coup veulent rejeter le monde tout court et parfois même la condition humaine et la vie, y compris la leur, en toute indifférence ou inconscience. Il y a sans doute là, plus que la marginalité, une recherche volontaire de la marginalité qui pourrait bien être la justification ultime de l'écriture.

J'ai dit au début que les personnages créés par les "cannibales» ne sont pas, pour la plupart, des victimes de la misère économique. Mais, violents, sans doute par mimétisme de la violence qu'ont prise les rapports humains 
et leur représentation par les médias, violents sans autre but que le plaisir du mal, non pas le mal métaphysique, mais le plaisir de faire mal, psychologiquement et physiquement, ils pourraient être une projection, déformée par le prisme du regard d'écrivains cultivés, d'une société où la révolte est, comme le suicide, un appel au secours.

\section{Notes}

1. N. Ammaniti, L. Brancaccio, A. Teodorani..., Gioventì cannibale, la prima antologia italiana dell'orrore estremo, a c. di D. Brolli, Torino, Einaudi, 1996.

2. Pour ce qui est de la place de ce courant dans l'historique des avant-gardes italiennes du XX⿳⺈ siècle, voir C. Farinato, I Cannibali : novità e tradizione in una scrittura di fine millennio, Maîtrise de littérature italienne contemporaine sous la direction de J.-Ch. Vegliante, UFR italien-roumain, Sorbonne Nouvelle- Paris 3, 2003, p. 3-5.

3. "Nella fortunata Gioventù cannibale [...] pochi superstiti» (R. Manica, Novecento, ultimo quarto, in L'ultima letteratura italiana, a c. di C. Lando e F. Pietrangeli, Roma, Manziana, 1999, p. 19).

4. N. Ammaniti, Io non ho paura, Torino, Einaudi, 2001. R. Manica estime qu' "Ammaniti a été un véritable cannibale». C. Lando, dans L'ultima letteratura italiana (op. cit., p. 35), précise que, à la différence d'autres "cannibales» comme A. Nove, il parle à la troisième personne, dans une perspective objective et que son récit reste compréhensible et vraisemblable. Pourtant, Fa un $p o$ ' male d'Ammaniti s'achève dans un monde de gitans, de prostitués des deux sexes, au bord d'une autoroute, aux abords de la ville, d'une marginalité tellement violente et caricaturée que cela frôle l'onirique et le fantastique.

5. Voir par exemple E Roma piange, de A. Teodorani et Cappuccetto Splatter, de D. Luttazzi, in Gioventù cannibale.

6. C. Lucarelli, Guernica, Torino, Einaudi, 2000.

7. John Frusciante est un guitariste du groupe Red Hot Chili Peppers. Les connaisseurs en font un disciple de Jimmy Hendricks.

8. E. Brizzi, Jack Frusciante è uscito dal gruppo, Ancona, Transeuropa, 1994. L'édition ici utilisée est celle de Baldini \& Castoldi, Milano, 1997.

9. Diminutif d'Adelaide. Mais Brizzi le dit d'entrée de jeu, c'est un clin d'œil vers la série télévisée Heidi, "la tipa dei cartoni animati che viveva nella baita svizzera" (Jack Frusciante ..., p. 23).

10. Voir S. Massaron, Il rumore, in Gioventù cannibale..., op. cit., p. 125-152.

11. Il faut évidemment faire une exception pour Guernica de C. Lucarelli.

12. D. Brolli, Le favole cambiano, introduction à Gioventù cannibale, p. V.

13. "quelli che non riuscivano a lavorare in nero finivano immancabilmente per farsi assumere dall'azienda più prospera e rigogliosa che si potesse trovare in posti come quello : la piccola criminalità organizzata» (S. Massaron, Il rumore, p. 127).

14. C. Farinato, I Cannibali..., p. 83-85.

15. F. Valabregue, Les mauvestis, Paris, POL, 2005.

16. In «le Monde des livres", 29 avril 2005.

17. Au moment où se situe la narration, «il veccchio Alex non aveva ancora compiuto diciott'anni» (Jack Frusciante..., p. 11); Brizzi lui-même en a à peine vingt au moment où paraît son livre.

18. Catholiques qui se reconnaissent dans le mouvement de "Communione e liberazione».

19. E. Brizzi, Jack Frusciante..., op. cit., p. 123. Le juge Falcone a été assassiné avec sa femme et leur escorte alors qu'ils se rendaient à l'aéroport de Palerme le 23 mai 1992. Il enquêtait sur la mafia, en particulier sur ses rapports avec certaines formations politiques et sur les fonds déposés en Suisse par cette organisation criminelle. Deux mois plus tard, le juge Borsellino était à son tour victime d'un attentat dans des conditions identiques. 


\section{QUELLES MARGINALITÉS?}

20. Il vit à Bologne mais ne se reconnaît pas dans la tradition politique de cette ville et de sa région.

21. In Superwoobinda, p. 68.

22. Woobinda est le héros éponyme d'une série télévisée australienne (39 épisodes) diffusée en Italie dans les années soixante-dix, ainsi qu'en France à partir de 1975. De son vrai nom Stevens, baptisé Woobinda par les aborigènes, il est vétérinaire et lutte pour sauver les animaux de la forêt australienne en butte aux attaques des braconniers.

23. In Woobinda, p. 15.

24. C. Farinato, I Cannibali..., p. 106.

25. E. Affinati, Secoli di gioventù, Milano, Mondadori, p. 18.

26. A. Nove, in Gioventù cannibale, p. 53-62.

27. A. Nove, Mia nonna, in Superwoobinda, p. 53-54.

28. Voir A. Nove, Il bagnoschiuma, in Superwoobinda: «Ho ammazzato i miei genitori perché usavano un bagnoschiuma assurdo [...] mia madre mi faceva schifo [...]. Mio padre diventava sempre più vecchio anche lui» (p. 7-8). Cette haine des parents est constamment présente dès le début de Jack Frusciante... où E. Brizzi l'étend avec commisération à la famille : «Questi poveri esseri umani costituivano, anni luce fa, una famiglia d'italiani viventi».

29. Où l'on trouve avec surprise "persino delle cose con attori che la nostra generazione aveva raramente sentito nominare : Jean Gabin, Louis De Funès, Peter Sellers» (Jack Frusciante..., p. 40).

30. Ibid.

31. Ibid., p. 44.

32. A. Nove, La frigidità dell'aria del mondo, in Superwoobinda, op. cit., p. 116.

33. Cf. C. Farinato, I Cannibali..., op. cit., p. 21-45.

34. "gli sembrava di conoscere Aidi da sempre, poiché quando si dice il sentimento ragazzi.» (Jack Frusciante..., p. 25)

35. D. Brolli, in Le favole..., op. cit. p. VIII.

36. Cf. C. Farinato, op. cit., p. 138-151.

37. Piero Cudini est mort brusquement le 5 novembre 2002. Le volume dans lequel il devait publier cette intervention n'a pas vu le jour.

38. A. Nove, La strage di via Palestro, in Superwoobinda, op. cit., p. 28.

39. Constante Girardengo (1893-1978). Sa carrière se déroule de 1913 à 1938. 